\title{
ATENÇÃO DOMICILIAR NA ESTRATÉGIA SAUUDE DA FAMÍLIA: PERSPECTIVAS DE IDOSOS, CUIDADORES E PROFISSIONAIS
}

\author{
Emanoel Avelar Muniz \\ Cibelly Aliny Siqueira Lima Freitas ${ }^{2}$ \\ Eliany Nazaré Oliveira ${ }^{3}$ \\ Maria Ribeiro Lacerda ${ }^{4}$
}

resumo

A Atenção Domiciliar (AD) é uma ação potente para ampliar a integralidade na Estratégia Saúde da Família (ESF) e torna-se necessária devido ao crescente número de idosos dependentes de cuidado no domicílio. Assim, objetivou-se analisar as perspectivas dos idosos,

\footnotetext{
1 Graduado em Enfermagem. Mestre em Saúde da Família pela Universidade Federal do Ceará (UFC) - Campus Sobral. Enfermeiro do Instituto Federal de Educação, Ciência e Tecnologia do Ceará (IFCE) - Campus Acaraú. E-mail: emanoelavelar@gmail.com.

2 Graduada em Enfermagem. Doutora em Enfermagem pela UFC. Docente do curso de Enfermagem da Universidade Estadual Vale do Acaraú (UVA) e do Mestrado Acadêmico em Saúde da Família (MASF) da UFC - Campus Sobral. E-mail: cibellyaliny@gmail.com.

3 Graduada em Enfermagem. Doutora em Enfermagem pela UFC. Docente do curso de Enfermagem da Universidade Estadual Vale do Acaraú (UVA) e do Mestrado Acadêmico em Saúde da Família (MASF) da UFC - Campus Sobral. E-mail: elianyy@hotmail.com.

4 Graduada em Enfermagem. Doutora em Enfermagem pela Universidade Federal de Santa Catarina (UFSC). Docente do Programa de Pós-graduação em Enfermagem da Universidade Federal do Paraná (UFPR). E-mail: mrlacerda55@gmail.com.
} 
familiares/cuidadores e profissionais da ESF sobre a AD. Esta pesquisa é de abordagem qualitativa do tipo exploratória, o campo de pesquisa foi a ESF do município de Sobral, Ceará. Os sujeitos do estudo foram 28 profissionais da ESF, 13 idosos e 13 familiares/cuidadores. A coleta de informações foi através de entrevista semiestruturada e grupo focal, que ocorreram entre setembro de 2014 e março de 2015; a análise se deu pela codificação temática. Foram identificados os tópicos semelhantes e específicos de cada grupo, emergindo-se quatro temáticas, a saber: a) percepções e sentimentos sobre a AD ao idoso na ESF; b) o apoio/suporte oferecido às famílias de idosos com comprometimento funcional pelas equipes da ESF; c) AD ao idoso na ESF: fatores facilitadores e limitantes; e d) as contribuições da estrutura física do domicílio para o cuidado do idoso dependente. Relatou-se a parceria da equipe multiprofissional, a fragilidade do apoio/suporte à família e a relação de dependência, na qual os papéis dos responsáveis pelo cuidado não estão bem definidos, sendo necessário potencializar a autonomia e corresponsabilização. Assim, identificou-se a necessidade de sensibilização dos profissionais e gestores sobre a relevância e especificidades do cuidado domiciliar ao idoso e cuidador, exigindo um perfil profissional e competências que devem ser desenvolvidas no ambiente de trabalho através de processos de educação permanente.

palavras-chave

Assistência Domiciliar. Saúde do Idoso. Estratégia Saúde da Família. Cuidadores. Pessoal de Saúde.

\section{Introdução}

O envelhecimento populacional é um fenômeno que vem sendo observado mundialmente e, no Brasil, o crescimento da população idosa merece atenção da sociedade. Segundo o último Censo Demográfico Brasileiro, em 2010, o contingente de indivíduos com idade igual ou superior a 60 anos ultrapassou os 20 milhões de pessoas, correspondendo a $11 \%$ da população; destes, $55,5 \%$ eram mulheres e 44,5\% homens (IBGE, 2010). Nesse sentido, reconhecendo a importância dos sistemas de saúde se prepararem para atender as necessidades da população que envelhece, a equipe de saúde da família deve estar organizada para acolher, além da demanda espontânea e programada, as particularidades 
de saúde da pessoa idosa com perdas funcionais e dependência para a realização das Atividades de Vida Diária (AVD), que necessitem de atendimento no domicílio (BRASIL, 2006).

Segundo Lacerda (2010), o cuidado domiciliar pode ser uma oportunidade significativa para que a autonomia do indivíduo e da família se concretize, já que o cuidado no domicílio do usuário, paciente/cliente e da família é uma ação e uma atitude. Afinal, mais do que um fazer, é um momento de vivenciar com o indivíduo e a família situações de saúde-doença, em seu lócus de habitação, de relações e de significado de vida.

Devem-se considerar também as especificidades inerentes à assistência domiciliar ao idoso. Primeiramente com relação ao espaço de cuidado que passa a ser o domicílio e, em segundo lugar, os atores envolvidos que tendem a ter uma relação mais duradoura e conflituosa já que envolve três entes responsáveis pelo processo saúde-adoecimento: o idoso, o familiar/cuidador e o profissional de saúde (VIEIRA et al., 2011).

Borges e Telles (2010) evidenciam a necessidade de desenvolver novas formas de apoio formal aos cuidadores familiares, exercidas por instituições públicas ou privadas. Além disso, também dar suporte aos profissionais da atenção básica, quando se deparam com os idosos que não possuem o suporte familiar ou não o recebem.

Devido à importância de aprofundar o conhecimento teórico nesse tema, visto o grande potencial da AD para ampliar a integralidade na ESF e provocar mudanças nas práticas dos profissionais e o grande número de idosos e familiares/cuidadores que não se sentem amparados pelo sistema/equipe de saúde, decorre a necessidade de refletir sobre como se dá o cuidado às famílias na AD de idosos com comprometimento funcional no contexto da ESF, a partir das perspectivas dos idosos, familiares/cuidadores e profissionais. Foram consideradas como perspectivas, de acordo com Flick (2009), as relações significativas com as quais a pessoa ou grupo lida com o tópico do estudo. Assim, objetivou-se analisar as perspectivas dos idosos, familiares/ cuidadores e profissionais da ESF sobre a Atenção Domiciliar realizada no âmbito da Estratégia Saúde da Família de Sobral, Ceará.

\section{Método}

Essa pesquisa é de abordagem qualitativa, do tipo exploratória. $\mathrm{O}$ campo de pesquisa foi o município de Sobral, principal cidade do noroeste do Ceará, localizada a 238 quilômetros de Fortaleza, contando com uma área de 
aproximadamente $2.123 \mathrm{~km}^{2}$ e uma população de 212.718 habitantes (SOBRAL, 2012). A ESF de Sobral está dividida em quatro macroáreas de saúde, de acordo com as características e proximidade dos territórios.

Diante disso, reconhecendo a grande quantidade e diversidade de territórios da ESF, foi utilizada a amostragem intencional, elegendo como critério de escolha a macroárea que possuía o maior número de idosos de acordo com o Sistema de Informação da Atenção Básica (SIAB) de 2013. Sendo assim, essa pesquisa foi realizada nos territórios da macroárea II, incluindo seis Centros de Saúde da Família da sede do município. A coleta de informações foi desenvolvida no período de setembro de 2014 a março de 2015.

Nesse estudo, escolheu-se a tríade profissional, idoso e cuidador/familiar por ser reconhecida a importância da mesma para a AD contínua e efetiva. Os sujeitos do estudo foram 28 profissionais da equipe mínima da ESF, incluindo 4 enfermeiras, 1 auxiliar de enfermagem e 23 Agentes Comunitários de Saúde (ACS). Destaca-se que os profissionais médicos não tiveram disponibilidade para participar nos momentos da coleta de informações.

Foram estabelecidos como critério de inclusão na pesquisa: os idosos que recebiam AD pelas equipes da ESF, com idade a partir de 60 anos; possuírem dificuldade ou impossibilidade física de locomoção até a unidade de saúde; apresentar dependência na realização das Atividades da Vida Diária (AVD), segundo índice de Katz; e capacidade cognitiva preservada de acordo com o Mini Exame do Estado Mental (MEEM), e também seus respectivos familiares/ cuidadores principais.

As escalas foram utilizadas para a escolha de sujeitos elegíveis para o estudo e para melhor caracterizá-los. O MEEM é um teste neuropsicológico para avaliação da função cognitiva. É rápido (dura em torno de 10 minutos), de fácil aplicação, não requerendo material específico. Avalia vários domínios (orientação temporal, espacial, memória imediata e de evocação, cálculo, linguagem-nomeação, repetição, compreensão, escrita e cópia de desenho). A pontuação total é de 30 pontos e a nota de corte sugerida para analfabetos é de 19 pontos (BRASIL, 2006). Destaca-se como fator limitante a baixa escolaridade dos idosos da pesquisa, o que contribuiu para a pequena pontuação no MEEM.

O Index de Independência nas AVD de Sidney Katz avalia a independência no desempenho de seis funções (banho, vestir-se, ir ao banheiro, transferência, continência e alimentação), classificando as pessoas idosas como dependentes ou independentes. Já a avaliação da sobrecarga dos cuidadores de Zarit serve para avaliar se os cuidadores de idosos estão sobrecarregados. Deve ser aplicada para o cuidador principal (pessoa que mais ajuda a pessoa idosa). A entrevista deverá ser realizada sem a presença da pessoa idosa (BRASIL, 2006). 
Assim, foram identificados 62 idosos; no entanto, 49 não foram incluídos na pesquisa e apenas 13 preencheram os critérios preestabelecidos. Foram excluídos da pesquisa os idosos considerados independentes de acordo com o Index de AVD, somando 24, e que possuíam comprometimento do estado mental de acordo com o MEEM, os quais foram identificados 34; 9 idosos foram excluídos por ambos os critérios.

Nesse sentido, pela importância de analisar as falas de ambas as partes, 13 familiares/cuidadores principais dos sessenta abordados foram incluídos, pois, se o idoso não preenchesse os critérios de inclusão, o familiar/cuidador automaticamente era excluído da pesquisa. Os sujeitos foram incluídos após consentirem com sua participação no estudo, de acordo com os princípios éticos norteadores das pesquisas envolvendo seres humanos.

Para a coleta de informações, utilizou-se a entrevista semiestruturada e o grupo focal. As entrevistas foram realizadas no domicílio dos idosos, identificados através da equipe de saúde da família, utilizando roteiro norteador que continha questões sobre aspectos sociodemográficos, problemas de saúde, uso de medicações, organização da atenção domiciliar, percepções sobre o cuidado domiciliar e a estrutura do domicílio. O tempo de duração das entrevistas ficou entre 15 e 20 minutos. Além das entrevistas, foram aplicadas aos idosos e cuidadores as escalas descritas anteriormente.

Também foram realizados 4 grupos focais com os profissionais em Centros de Saúde da Família (CSF) diferentes, na oportunidade da roda (espaço de reunião da equipe), 1 encontro por CSF, de 30 a 40 minutos, contando com 6 a 9 participantes, onde foram escolhidos aleatoriamente e de acordo com a disponibilidade dos profissionais representando as diversas categorias da equipe mínima da ESF, utilizando-se um roteiro de questões norteadoras abordando o apoio/suporte as famílias de idosos dependentes, fatores facilitadores e limitantes da $\mathrm{AD}$, estrutura dos domicílios, estímulo à autonomia e contribuições ao processo saúde-doença. Destaca-se que em 2 CSFs não foi possível realizar o grupo focal: em um, a unidade de saúde encontrava-se em reforma, e no outro houve dificuldade de realizar o agendamento com a equipe.

A proposta de análise do material escolhida foi a codificação temática. Segundo Flick (2009), esse procedimento foi desenvolvido para estudos comparativos, nos quais os grupos em estudo são provenientes da questão de pesquisa, sendo, assim, definidos a priori. A questão da pesquisa é a distribuição social de perspectivas sobre um fenômeno ou um processo. A amostragem é voltada aos grupos cujas perspectivas sobre a questão pareçam ser mais instrutivas para a análise, e que são, portanto, definidos antecipadamente. 
Assim, as falas dos sujeitos foram gravadas e transcritas na íntegra, e então foram construídas as perspectivas dos idosos, familiares/cuidadores e profissionais sobre a Atenção Domiciliar realizada no âmbito da Estratégia Saúde da Família, através da análise dos consensos, conflitos e contradições presentes no modo como a AD vem sendo operacionalizada na ESF de Sobral. Para isso, adotaram-se as recomendações éticas para pesquisas envolvendo seres humanos, obtendo a aprovação no Comitê de Ética em Pesquisa da Universidade Estadual Vale do Acaraú (n. ${ }^{\circ}$ do Certificado de Apresentação para Apreciação Ética (CAEE) = 31215114.9.0000.5053). Este artigo é um recorte da pesquisa de dissertação intitulada "Atenção Domiciliar na Estratégia Saúde da Família: análise das perspectivas de idosos, cuidadores e profissionais", apresentada ao Mestrado Acadêmico em Saúde da Família da Universidade Federal do Ceará - Campus Sobral.

\section{Resultados e discussão}

As características sociodemográficas dos 13 idosos incluídos no estudo são: idosos de todas as idades, incluindo mais de 80 anos (4); paridade entre os sexos (7 feminino e 6 masculino); a maioria (11) eram analfabetos, de cor parda (10) e religião católica (10); quanto ao estado civil, eram casados (5), viúvos (5), separados (2) e solteiro (1). Em relação aos 13 familiares/cuidadores da pesquisa: possuem a partir de 30 anos de idade, inclusive 1 com mais de 60 anos; maioria do sexo feminino (12); baixa escolaridade (6 possuíam 1 a 4 anos de estudo); raça parda (10); religião católica (10); estado civil solteiro (8); e apresentam sobrecarga leve (10), de acordo com a escala de Zarit.

A partir da comparação das perspectivas dos idosos, familiares/cuidadores e profissionais da ESF, foram identificados os tópicos semelhantes e específicos de cada grupo, emergindo, então, quatro temáticas, a saber: a) percepções e sentimentos sobre a Atenção Domiciliar ao idoso na ESF; b) o apoio/suporte oferecido às famílias de idosos com comprometimento funcional pelas equipes da ESF; c) a atenção Domiciliar ao idoso na ESF: fatores facilitadores e limitantes; e d) as contribuições da estrutura física do domicílio para o cuidado do idoso. A seguir encontra-se o Quadro 1, com a síntese dessas perspectivas contendo os assuntos principais. 
Quadro 1 - Comparação das perspectivas de idosos, familiares/cuidadores e profissionais sobre a Atenção Domiciliar na Estratégia Saúde da Família em Sobral, Ceará, 2015.

\begin{tabular}{|c|c|c|c|}
\hline Perspectiva & Idoso & Cuidador & Profissional \\
\hline $\begin{array}{l}\text { Percepções } \\
\text { e sentimentos } \\
\text { sobre a AD }\end{array}$ & $\begin{array}{l}\text { - Indecisão/confusão } \\
\text { - Desaprovação } \\
\text { - Satisfação } \\
\text { - Impotência } \\
\text { - Inexistência de } \\
\text { cuidado } \\
\text { - Conformismo } \\
\text { - Atendimento parcial } \\
\text { das necessidades }\end{array}$ & $\begin{array}{l}\text { - Divergências } \\
\text { - Satisfação } \\
\text { - Desvalorização } \\
\text { - Felicidade } \\
\text { - Agradecimento } \\
\text { - Conforto } \\
\text { - Segurança } \\
\text { - Importância } \\
\text { - Impotência }\end{array}$ & $\begin{array}{l}\text { - Dependência } \\
\text { - Abandono/negligência } \\
\text { - Maus-tratos } \\
\text { - Estresse } \\
\text { - Angústia } \\
\text { - Impotência } \\
\text { - Ausência de empatia } \\
\text { - Juízo de valor negativo } \\
\text { - Progresso }\end{array}$ \\
\hline $\begin{array}{l}\text { Ações de } \\
\text { apoio/suporte } \\
\text { às famílias }\end{array}$ & - Não reconhece & $\begin{array}{l}\text { - Acesso a } \\
\text { medicamentos } \\
\text { - Agendamento } \\
\text { de consultas e exames } \\
\text { - Não reconhece } \\
\text { - Encaminhamentos } \\
\text { para outros serviços }\end{array}$ & $\begin{array}{l}\text { - Acesso a medicamentos } \\
\text { - Agendamento de consul- } \\
\text { tas e exames } \\
\text { - Conversas/orientações } \\
\text { - Distribuição de tarefas } \\
\text { - Atenção psicossocial } \\
\text { - Projetos/grupos }\end{array}$ \\
\hline $\begin{array}{l}\text { Fatores } \\
\text { facilitadores }\end{array}$ & $\begin{array}{l}\text { - Melhoria das condi- } \\
\text { ções de saúde } \\
\text { - Acompanhamento } \\
\text { e avaliação da saúde } \\
\text { - Verbalização de } \\
\text { problemas } \\
\text { - Comodidade }\end{array}$ & $\begin{array}{l}\text { - Acompanhamento } \\
\text { do ACS } \\
\text { - Acolhimento } \\
\text { dos profissionais }\end{array}$ & $\begin{array}{l}\text { - Núcleo de Apoio a } \\
\text { Saúde da Família (NASF) } \\
\text { - Residências } \\
\text { multiprofissionais } \\
\text { - Disponibilidade de medi- } \\
\text { camentos e exames } \\
\text { - Garantia de transporte } \\
\text { - Abertura da gestão } \\
\text { - Interesse e presença } \\
\text { do ACS }\end{array}$ \\
\hline $\begin{array}{l}\text { Fatores } \\
\text { limitantes }\end{array}$ & $\begin{array}{l}\text { - Assistencialismo } \\
\text { - Falta de } \\
\text { medicamentos } \\
\text { - Acompanhamento } \\
\text { não sistemático } \\
\text { - Desinteresse dos } \\
\text { profissionais }\end{array}$ & $\begin{array}{l}\text { - Acompanhamento } \\
\text { não sistemático } \\
\text { - Ausência do médico } \\
\text { - Falta de medicamentos } \\
\text { - Grande demanda } \\
\text { - Falta de tempo e inte- } \\
\text { resse dos profissionais } \\
\text { - Falta de transporte } \\
\text { - Dificuldade de acesso }\end{array}$ & $\begin{array}{l}\text { - Falta de materiais, medi- } \\
\text { camentos e transportes } \\
\text { - Comodismo do idoso e } \\
\text { família } \\
\text { - Despreparo dos } \\
\text { cuidadores } \\
\text { - Aspectos culturais } \\
\text { - Maus-tratos/violência } \\
\text { - Sobrecarga do cuidador } \\
\text { - Idosos vivendo sozinhos } \\
\text { - Grande demanda }\end{array}$ \\
\hline $\begin{array}{l}\text { Estrutura do } \\
\text { domicílio }\end{array}$ & $\begin{array}{l}\text { - Pequeno espaço } \\
\text { físico } \\
\text { - Precárias condições } \\
\text { de ventilação } \\
\text { - Barreiras físicas } \\
\text { - Satisfação }\end{array}$ & $\begin{array}{l}\text { - Barreiras físicas } \\
\text { - Falta de adaptações } \\
\text { - Precária acessibilidade } \\
\text { da comunidade }\end{array}$ & $\begin{array}{l}\text { - Espaço limitado } \\
\text { - Piso esburacado } \\
\text { - Acesso difícil } \\
\text { - Precárias condições de } \\
\text { higiene e ventilação } \\
\text { - Criação de animais }\end{array}$ \\
\hline
\end{tabular}

Fonte: Dados da pesquisa. 
Diante do exposto, identificam-se divergências nos discursos dos idosos, cuidadores e profissionais nas suas percepções e sentimentos relacionados à $\mathrm{AD}$, variando da inexistência de cuidado, atendimento parcial das necessidades até satisfação, agradecimento e progresso no estado de saúde. Chama atenção o sentimento de impotência compartilhado pelos três grupos, demonstrando a complexidade e os desafios do trabalho em AD.

Nesse sentido, Gomes et al. (2012) informam que sujeito, família e profissionais podem ser consideradas unidades complexas que, ao se articularem e organizarem, formam um sistema a ser compreendido na relação que mantêm com o ambiente e o tempo. Nesse ínterim, a equipe atua como apoio às famílias, instrumentalizando-as para o cuidar.

No que se refere às ações de apoio e suporte oferecidas às famílias de idosos com comprometimento funcional pelas equipes da ESF, o grupo de idosos e parte dos cuidadores não reconheceu nenhuma, evidenciado a necessidade de potencializar estratégias e ferramentas nesse sentido. Alguns cuidadores e profissionais destacaram a facilitação do acesso a medicações de uso contínuo e a marcação de consultas e exames como formas de ajuda. No discurso dos profissionais, foram incluídas as conversas, orientações, atenção psicossocial, disponibilização de alimentos, aparelhos e materiais, a realização de grupos e projetos.

Diante disso, o Ministério da Saúde (BRASIL, 2012) afirma que as redes formais de apoio ao idoso/família são frágeis, pois é visível a ausência de relações e interações que se conectem de forma integrada para gerar um trabalho conjunto. Fica evidente que o cuidado é somente para o idoso, ficando o familiar cuidador excluído deste processo.

Com relação aos fatores positivos da $\mathrm{AD}$, cada grupo apresentou especificidades. Enquanto os idosos referiram melhora nas suas condições de saúde por conta do acompanhamento e avaliação dos profissionais, possibilidade de verbalização de problemas e a comodidade de receber o cuidado no domicílio pela equipe da ESF; os cuidadores destacaram o acompanhamento frequente dos ACS e o acolhimento realizado pelos profissionais de suas queixas e dúvidas.

Os profissionais, por sua vez, elencaram o apoio do NASF e das residências multiprofissionais, a disponibilidade de medicamentos, exames e profissionais, a garantia de transporte, a abertura da gestão, a integração da equipe, etc. Ganhou destaque também a importância do prazer pelo trabalho, da articulação intersetorial e da condição financeira da família para a construção de um cuidado domiciliar de qualidade e humanizado. Um aspecto positivo da $\mathrm{AD}$ compartilhado pelos cuidadores e profissionais é o interesse e a presença constante no domicílio do ACS.

Nesse sentido, Motta, Aguiar e Caldas (2011) destacam a centralidade do trabalho dos ACS para a atenção aos idosos. Sua função inclui cadastrar, 
acompanhar as famílias e intermediar a relação entre idosos, famílias e equipes. É sua atribuição registrar os idosos que foram hospitalizados e/ou regressaram de internação hospitalar, e identificar aqueles que necessitam de assistência domiciliar, mediante critérios acordados localmente pelas equipes.

Dentre os fatores limitantes da $\mathrm{AD}$, os três grupos de sujeitos relataram a falta de medicamentos; o acompanhamento não sistemático dos idosos e das famílias, ocasionado muitas vezes pela desorganização da equipe; o desinteresse de alguns profissionais e a desvalorização da AD ao idoso pela ESF; a grande demanda de trabalho nas unidades de saúde; e a falta de transportes frequente. Nesse ponto de vista, Carvalhais e Sousa (2013) sugerem que o principal obstáculo para a realização de cuidados domiciliares de qualidade é a escassez de recursos e a desorganização traduzida em falta de tempo, desordem e frustração. Nesse sentido, Lacerda (2010) insiste que a preparação, o ter atitudes, o aceitar o desafio que cuidar nas residências apresenta e o querer fazer são imprescindíveis para que o profissional realize o cuidado domiciliar.

Os profissionais de saúde destacaram como entraves para a realização do cuidado ao idoso no domicílio o comodismo do próprio idoso e da família, a desconfiança do idoso no cuidado prestado pelos familiares, o despreparo dos cuidadores e a sobrecarga de trabalho destes. Foram frequentes os relatos de violência ao idoso, incluindo negligência e maus-tratos. Esses casos merecem um olhar vigilante da equipe e o acionamento de serviços de proteção quando necessário. Borges e Telles (2010) também destacaram as interações familiares negativas com referências aos maus-tratos com os idosos como um dos fatores limitantes do cuidado domiciliar.

De acordo com os idosos, cuidadores e profissionais, alguns domicílios apresentam barreiras físicas e ausência de adaptações que permitam ao idoso com comprometimento funcional viver com o máximo possível de autonomia. Em estudo realizado por Gitlin et al. (2014) com pacientes portadores de demência, encontrou-se que a maioria deles morava em casas com alto risco de queda, enfrentando desafios ambientais e vários perigos. Os resultados sugerem a importância de melhorar a qualidade de vida, abordando fatores de risco modificáveis e o planejamento de intervenções para o paciente, considerando as perspectivas do cuidador.

Goyanna et al. (2013) também encontraram fragilidades quanto à adaptação dos domicílios às necessidades do idoso, mesmo reconhecendo que existe um esforço dos familiares quanto ao ajuste desse espaço. Nesse contexto, muitas vezes a condição socioeconômica é fator limitante para a realização de tais mudanças. A população idosa, especialmente aquela com poucos recursos econômicos, tem necessidades substanciais de cuidados contínuos. Assim, 
políticas para melhorar os serviços de suporte a longo prazo e reduzir a necessidade não atendida podem beneficiar os idosos e aqueles que cuidam deles (FREEDMAN; SPILLMAN, 2014).

Diante do exposto, é necessário compreender as especificidades do cuidado domiciliar, no qual o domicílio se insere como espaço de atenção à saúde e local privado das pessoas, onde o profissional tem que pedir licença para entrar, além da complexidade da execução da AD na ESF por conta da diversidade de ações programáticas, grande demanda, falta de estrutura dos serviços e profissionais despreparados para atender no domicílio, mas que possuem essa responsabilidade.

\section{Considerações finais}

Essa pesquisa proporcionou uma análise da AD ao idoso com comprometimento funcional para o sistema local de saúde e trouxe algumas contribuições para a ESF. A necessidade de sensibilização dos profissionais e gestores sobre a relevância e especificidades do cuidado domiciliar ao idoso e cuidador, exigindo um perfil profissional e competências que devem ser desenvolvidas no ambiente de trabalho através de processos de educação permanente, são algumas delas.

Diferentes percepções e sentimentos sobre a AD foram observados entre os sujeitos envolvidos. Nesse sentido, a relação entre profissional, idoso e família assume um papel central em todo o processo de cuidado. Outra característica presente foi a dinamização de parcerias entre a equipe multiprofissional através da comunicação e contribuição das categorias para o plano de cuidados.

Assim, a ESF de Sobral apresenta algumas potencialidades, como a boa relação entre os profissionais e uma preocupação com a autonomia e a independência do idoso. Todavia, é preciso que a família seja capacitada com o apoio do sistema de saúde através de um acompanhamento sistemático, no qual a promoção da qualidade de vida do idoso e da família seja estabelecida como política pública de saúde. Uma iniciativa que pode ser indicada é a formação de grupos de cuidadores dos idosos sob acompanhamento da equipe multiprofissional.

Como limitação do estudo, identificou-se a pequena verbalização dos idosos e dos cuidadores e a não participação da categoria médica durante a coleta de dados, podendo contribuir para uma análise mais abrangente da questão de pesquisa. É importante a continuidade de pesquisas sobre esta temática, envolvendo também outras características de idosos e cuidadores, além das perspectivas dos profissionais do NASF e dos Serviços de Atenção Domiciliar, como o Programa Melhor em Casa. 
HOME CARE IN THE FAMILY HEALTH

STRATEGY: PERSPECTIVES OF THE ELDERLY, CAREGIVERS AND PROFESSIONALS

abstract

The Home Care $(\mathrm{HC})$ is a powerful action to increase the integrality in the Family Health Strategy (FHS), and is necessary due to the increasing number of elderly people dependent on care at home. Thus, the objective was to analyze the perspectives of the elderly, family/caregivers, and FHS professionals about HC. This research has a qualitative exploratory approach, the field of research was the FHC of the city of Sobral, Ceará. The subjects of the study were twenty eight ESF professionals, thirteen elderly, and thirteen family members/caregivers. The data was collected through semi-structured interviews and focus groups, and occurred between September 2014 and March 2015; the analysis was based on thematic coding. The similar and specific topics to each group were identified, leading to the emergence of four themes, namely: a) perceptions and feelings about HC to the elderly in FHS; b) the support/backup offered to the families of elderly people with functional impairment by the FHS teams; c) HC to elderly in FHS: facilitating and limiting factors; and d) the contributions of the infrastructure of the home to the care of the dependent elderly. The research results reported the partnership of the multiprofessional team, the fragility of support/backup to the family and the relationship of dependence, in which the roles of those responsible for care are not well defined and it becomes necessary to enhance autonomy and co-responsibility. Thus, it was possible to identify the need to sensitize professionals and managers about the relevance and specificities of home care to the elderly and caregivers, requiring a professional profile and skills that must be developed in the work environment through permanent education processes.

keywords

Home Nursing. Health of the Elderly. Family Health Strategy. Caregivers. Health Personnel. 
BORGES, Maria Marta de Castro; TELLES, José Luiz. O cuidado do idoso no contexto familiar: percepção da equipe de saúde da família. Revista Brasileira de Geriatria e Gerontologia, Rio de Janeiro, v. 13, n. 3, p. 349-360, dez. 2010. Disponível em: <http:// www.scielo.br/pdf/rbgg/v13n3/a02v13n3.pdf>. Acesso em: 12 jun. 2018.

BRASIL. Ministério da Saúde. Secretaria de Atenção à Saúde. Departamento de Atenção Básica. Caderno de atenção domiciliar. Brasília: Ministério da Saúde, 2012. v. 1.

. Ministério da Saúde. Secretaria de Atenção à Saúde. Departamento de Atenção Básica. Envelhecimento e saúde da pessoa idosa. Brasília: Ministério da Saúde, 2006.

CARVALHAIS, Maribel; SOUSA, Liliana. Qualidade dos cuidados domiciliares de enfermagem a idosos dependentes. Saúde e Sociedade, São Paulo, v. 22, n. 1, p. 160-172, 2013. Disponível em: <http://www.scielo.br/pdf/sausoc/v22n1/15.pdf>. Acesso em: 7 jun. 2015.

FLICK, Uwe. Introdução à pesquisa qualitativa. Tradução Joice Elias Costa. 3. ed. Porto Alegre: Artmed, 2009. 405 p.

FREEDMAN, Vicki; SPILLMAN, Brenda. Disability and care needs among older Americans. A Multidisciplinary Journal of Population Health and Health Policy, New York, v. 92, n. 3, p. 509-540, 2014. Disponível em: <http://onlinelibrary.wiley.com/doi/10.1111/14680009.12076/>. Acesso em: 26 jul. 2015.

GITLIN, Laura et al. Correlates of quality of life for individuals with dementia living at home: the role of home environment, caregiver, and patient-related characteristics. The American Journal of Geriatric Psychiatry, Philadelphia, v. 22, n. 6, p. 587-597, June 2014. Disponível em: <http://www.ncbi.nlm.nih.gov/pubmed/23890928>. Acesso em: 30 nov. 2015.

GOMES, Ingrid Meireles et al. Aspectos éticos nas redes sociais de apoio no cuidado domiciliar à luz do pensamento complexo. Enfermagem em Foco, Brasília, v. 3, n. 3, p. 110-113, 2012. Disponível em: <http://revista.portalcofen.gov.br/index.php/enfermagem/article/view/292>. Acesso em: 5 jun. 2015.

GOYANNA, Natália Frota et al. Idosos com doença de Alzheimer: como vivem e percebem a atenção na Estratégia Saúde da Família. Revista Cuidado É Fundamental, Rio de Janeiro, v. 9, n. 2, p. 379-386, 2017. Disponível em: <http://www.seer.unirio.br/index. php/cuidadofundamental/article/view/5037/pdf>. Acesso em: 9 jun. 2017.

INSTITUTO BRASILEIRO DE GEOGRAFIA E ESTATÍSTICA (IBGE). Ministério do Planejamento, Orçamento e Gestão. Censo demográfico 2010: características gerais da população, religião e pessoas com deficiência. Rio de Janeiro: IBGE, 2010.

LACERDA, Maria Ribeiro. Cuidado domiciliar: em busca da autonomia do indivíduo e da família na perspectiva da área pública. Ciência e saúde coletiva, Rio de Janeiro, v. 15, n. 5, p. 2621-2626, ago. 2010. Disponível em: <http://www.scielo.br/scielo. php?script=sci_arttext\&pid=\$1413-81232010000500036>. Acesso em: 12 jun. 2018.

MOTTA, Luciana Branco da; AGUIAR, Adriana Cavalcanti de; CALDAS, Célia Pereira. Estratégia Saúde da Família e a atenção ao idoso: experiências em três municípios brasileiros. Cadernos de Saúde Pública, Rio de Janeiro, v. 27, n. 4, p. 779-786, abr. 2011. Disponível em: <http://www.scielo.br/scielo.php?script=sci_arttext\&pid=S0102311X2011000400017>. Acesso em: 12 jun. 2018.

SOBRAL (Município). Prefeitura Municipal. Site da Prefeitura de Sobral. Sobral, 2012. Disponível em: <http://www.sobral.ce.gov.br>. Acesso em: 1 nov. 2012. 
VIEIRA, Chrystiany Plácido de Brito et al. Práticas do cuidador informal do idoso no domicílio. Revista Brasileira de Enfermagem, Brasília, v. 64, n. 3, p. 570-579, jun. 2011. Disponível em: <http://www.scielo.br/scielo.php?script=sci_arttext\&pi $d=$ S0034-71672011000300023 >. Acesso em: 12 jun. 2018.

Data de Submissão: 28/12/2015

Data de Aprovação: 08/05/2018 